\title{
Phytochemical and pharmacology effect of Panax notoginseng
}

\author{
Bui Thanh Tung*, Nguyen Thanh Hai \\ School of Medicine and Pharmacy, Vietnam National University, Office 506, Building Y1, 144 Xuan Thuy, Cau Giay, Ha Noi, Vietn am.
}

\section{ARTICLE INFO \\ Article history: \\ Received on: 01/07/2016 \\ Revised on: 22/07/2016 \\ Accepted on: 11/08/2016 \\ Available online: 30/08/2016}

\section{Key words:}

Panax notoginseng,

antioxidant,

antiinflammation,

antithrombotic,

phytochemical.

\begin{abstract}
Panax notoginseng has been shown beneficial effects on the cardiovascular system and traditionally used to prevent cerebral ischemia. It has haemostatic, antioxidant, hypolipidaemic, hepatoprotective, renoprotective and estrogen-like activities. The phytochemical compositions of the Panax notoginseng are mainly saponins, which belong to protopanaxatriol and protopanaxadiol group. Furthermore, Panax notoginseng also has some compounds such as notoginsenoide, flavonoids, phytosteroid. The present review focuses on phytochemical and important pharmacology effect of Panax notoginseng including antioxidant, antiinflammation and antithrombotic activities.
\end{abstract}

\section{INTRODUCTION}

Panax notoginseng (Burk.) F. H. Chen (Araliaceae) is a medicinal plant. It is distributed throughout the southwest of China, Japan, Burma, Nepal and Vietnam. This plant belongs to the same family and genus as $P$. ginseng. The root of Panax notoginseng is normally harvested in autumn before flowering, after three years of growth. After harvesting, the roots are cleaned, divided into main root, branch root and rhizome then dried under the sun or with artificial heating at low temperatures (Wang et al., 2008). Panax notoginseng has sweet and slightly bitter in flavour, slightly warm in nature. This plant is usually applied to treat various kinds of bleeding and pains due to blood stasis (Yang et al., 2014). The figure 1 shows the whole plant and root of Panax notoginseng. In this review we summarize some

\footnotetext{
* Corresponding Author

Bui Thanh Tung, School of Medicine and Pharmacy, Vietnam National University, Office 506, Building Y1, 144 Xuan Thuy, Cau Giay, Ha Noi, Vietnam.Email:tungasia82@yahoo.es
}

characteristics of phytochemical and pharmacology effect of the Panax notoginseng.

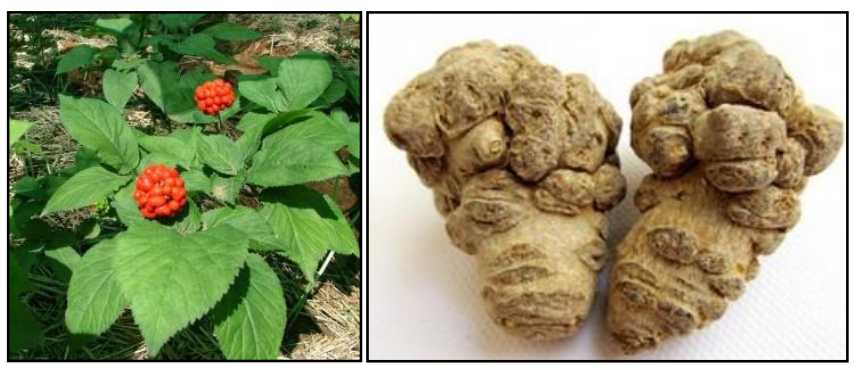

Fig. 1: Whole plant and root of Panax notoginseng

\section{Chemical constituents of Panax notoginseng}

The phytochemical constituents of Panax notoginseng are complex, consisting of various saponins, amino acids, polyacetylenes, volatile oils, polysaccharides and flavonoids. The dammarane-type saponins achieves to $12 \%$ of the total root content. These are the main bioactive compounds of the root with many beneficial pharmacology effects. 
Panax notoginseng has higher percentage of total saponins compared to $P$. ginseng and $P$. quinquefolium. Its saponins compose various ginsenosides and notoginsenosides. Many of these ginsenosides in the root are also presented in other Panax species such as $P$. ginseng and $P$. quinquefolium. Actually, there are more than 30 ginsenosides which have been isolated. The saponins are classified into three main classes based on their aglycones - protopanaxadiol (e.g. ginsenosides Rb1, Rb2, Rc, Rd), protopanaxatriol (e.g. ginsenosides $\mathrm{Rg} 1, \mathrm{Re}, \mathrm{Rf}$ ) and oleanolic acid (e.g. ginsenoside Ro) (Kim, 2012). Other group of saponins, notoginsenosides, including notoginsenosides R1 to R4, R6 to R9 notoginsenosides $\mathrm{A}$ to $\mathrm{N}$, notoginsenosides $\mathrm{Fa}, \mathrm{Fc}$ and $\mathrm{Fe}$, have been isolated from Panax notoginseng (Yoshikawa et al., 2001). Some of saponins in Panax notoginseng are presented in figure 2.

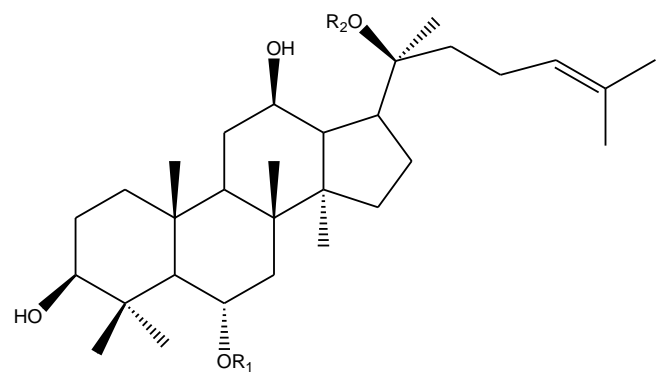

Protopanaxatriol $\left(\mathrm{R}_{1}, \mathrm{R}_{2}=\mathrm{H}\right)$

\begin{tabular}{lll} 
Protopanaxatriol $\left(\mathrm{R}_{1}, \mathrm{R}_{2}=\mathrm{H}\right)$ & & \\
\hline Saponins & $\mathrm{R}_{1}$ & $\mathrm{R}_{2}$ \\
Ginsenoside Rgl & $-\mathrm{Glc}$ & $-\mathrm{Glc}$ \\
Ginsenoside Re & $-\mathrm{Glc}^{2}-{ }^{1} \mathrm{Rha}$ & $-\mathrm{Glc}$ \\
Ginsenoside Rf & $-\mathrm{Glc}^{2}-{ }^{1} \mathrm{Glc}$ & $-\mathrm{H}$ \\
Notoginsenoside $\mathrm{R}_{1}$ & $-\mathrm{Glc}^{2}-{ }^{1} \mathrm{Xyl}$ & $-\mathrm{Glc}$ \\
Notoginsenoside $\mathrm{R}_{2}$ & $-\mathrm{Glc}^{2}-{ }^{1} \mathrm{Xyl}$ & $-\mathrm{H}$ \\
Notoginse noside $\mathrm{R}_{3}$ & $-\mathrm{Glc}$ & $-\mathrm{Glc}^{6}-{ }^{1} \mathrm{Glc}$ \\
Notoginsenoside $\mathrm{R}_{6}$ & $-\mathrm{Glc}$ & $-\mathrm{Glc}^{6}-{ }^{1} \mathrm{Glc}(\alpha)$ \\
\hline
\end{tabular}

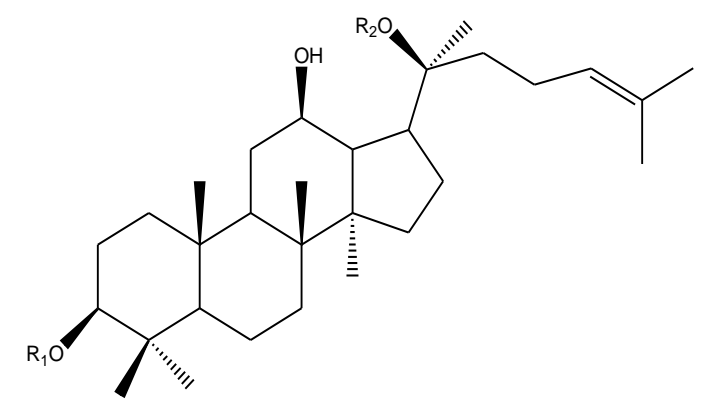

Protopanaxadiol $\left(\mathrm{R}_{1}, \mathrm{R}_{2}=\mathrm{H}\right)$

\begin{tabular}{|c|c|c|}
\hline Saponins & $\mathrm{R}_{1}$ & $\mathrm{R}_{2}$ \\
\hline Ginsenoside $\mathrm{Rbl}$ & $-\mathrm{Glc}^{2}-{ }^{1} \mathrm{Glc}$ & $-\mathrm{Glc}^{6}-{ }^{1} \mathrm{Glc}$ \\
\hline Ginsenoside Rb2 & $-\mathrm{Glc}^{2}-{ }^{1} \mathrm{Glc}$ & $-\mathrm{Glc}^{6}-{ }^{1} \mathrm{Ara}(\mathrm{p})$ \\
\hline Ginsenoside Rb3 & $-\mathrm{Glc}^{2}-{ }^{1} \mathrm{Glc}$ & $-\mathrm{Glc}^{6}-{ }^{1} \mathrm{Xyl}(\mathrm{p})$ \\
\hline Ginsenoside Rc & $-\mathrm{Glc}^{2}-{ }^{1} \mathrm{Glc}$ & $-\mathrm{Glc}^{6}-{ }^{1} \mathrm{Ara}(\mathrm{f})$ \\
\hline Ginsenoside Rd & $-\mathrm{Glc}^{2}-{ }^{1} \mathrm{Glc}$ & -Glc \\
\hline Notoginsenoside $\mathrm{R}_{4}$ & $-\mathrm{Glc}^{2}-{ }^{1} \mathrm{Glc}$ & $-\mathrm{Glc}^{6}-{ }^{1} \mathrm{Glc}^{6}-{ }^{1} \mathrm{Xyl}$ \\
\hline Notoginsenoside $\mathrm{Fa}$ & $-\mathrm{Glc}^{2}-{ }^{1} \mathrm{Glc}^{2}-{ }^{1} \mathrm{Xyl}$ & $-\mathrm{Glc}^{6}-{ }^{1} \mathrm{Glc}$ \\
\hline Notoginsenoside Fc & $-\mathrm{Glc}^{2}-{ }^{1} \mathrm{Glc}^{2}-{ }^{1} \mathrm{Xyl}$ & $-\mathrm{Glc}^{6}{ }_{-1}^{1} \mathrm{Xyl}$ \\
\hline Notoginsenoside Fe & -Glc & $-\mathrm{Glc}^{6}{ }^{1} \mathrm{Ara}(\mathrm{f})$ \\
\hline
\end{tabular}

Fig. 2: Chemical structures of some saponins. Abbreviations: Glc, glucose; $\operatorname{Ara}(\mathrm{f})$, arabinose in furanose form; $\operatorname{Ara}(\mathrm{p})$, arabinose in pyranose form; Rha, rhamnose; Xyl, xylose (Jiang, 2007)
Dencichine ( $\beta$-N-oxalyl-L- $\alpha, \beta$-diaminopropionic acid) is an important and bioactive amino acid and presented in Panax notoginseng. It has strong haemostatic activity (Long et al., 1996). Panax notoginseng also presents some types of amino acids, such as aspartic acid, glutamic acid, arginine, lysine and leucine. The total amount of amino acids arises about $7.73 \%$. Trilinolein, a triacylglycerol with three linoleate residues, is another important compound that was found. It has protective effects against cardiovascular diseases by reducing thrombogenicity, arrhythmias, increasing erythrocyte deformability and counteract free radical damages (Chan et al., 2002). Some polyacetylenes including panaxynol, panaxydol and panaxytriol have also been isolated in Panax notoginseng. These compounds have showed cytotoxic, antiplatelet and anti inflammatory activities. Other compounds such as, notoginsenic acid betasophoroside, was isolated also from the roots. Various polysaccharides, such as sanchinan-A, arabinogalactan, were isolated from Panax notoginseng and have strong immunostimulatory effects (Yoshikawa et al., 1997). A small chitinase-like antifungal protein whose molecular weight of $15 \mathrm{kDa}$ was found in the root of this plant. Moreover, Panax notoginseng contains a series of sesquiterpenes essential oils and some of them have been identified as $\alpha$-guaiene, $\beta$-guaiene, and octadecane (Zhu et al., 2005). Some flavonoids including quercetin, kaempferol, $\beta$-sitosterol, stigmasterol, daucosterol also are presented in this medicinal plant (Wang et al., 2006).

\section{Pharmacological activities of Panax notoginseng}

The Panax notoginseng have many different traditional uses. The raw form is officially recognized in Chinese medicine for its haemostatic and cardiovascular properties, such as arrest bleeding, disperse blood clots, improve blood circulation, disperse bruises, cause subsidence of swelling and alleviate pain.

Its well-known haemostatic effect has been studied scientifically (Fan et al., 2005). Several beneficial cardiovascular effects were shown. Animal studies have shown that Panax notoginseng extracts decreased blood pressure and peripheral vascular resistance. It increases coronary blood flow, treats experimental myocardial ischemia, alleviates angina pectoris, increases cardiac contractility and, may have anti-arrhythmic effects as well as helps in craniocerebral trauma (Wang et al., 2006). Other reported pharmacological activities of raw Panax notoginseng extracts and its total saponins include reduction of blood viscosity, inhibition of platelet aggregations (Wang et al., 2004), anti-inflammatory and analgesic effects (Rhule et al., 2006), retardation of the progress of early diabetic nephropathy, immunological adjuvant activities (Sun et al., 2006), prevention of liver fibrosis and hepatic microvascular dysfunction (Park et al., 2005). Furthermore, the anti-carcinogenic and anti-proliferative activities of the roots of Panax notoginseng have been reported (Lam et al., 2001). Notoginsenoside R1, one of the distinct saponins which were presented in Panax notoginseng, has been found. It helps increase the fibrinolytic potential in cultured endothelial and smooth muscle cells, counteract endotoxin- 
induced activation of endothelial cells and to inhibit TNF-alphainduced PAI-1 production in human aortic smooth muscle cells (Zhang et al., 2006).

\section{Antioxidant activity}

Reactive oxygen species (ROS), such as superoxide anion, hydroxyl radicals and hydrogen peroxide, are chemically reactive molecules derived from oxygen. They are generated in living organisms as by-products through many metabolic pathways. ROS can readily react with and oxidize most biomolecular including carbohydrates, proteins, lipids and DNA whereby increase evidence that accumulation of ROS in biological system causes oxidative damage to tissue that affects cellular integrity and functions. Oxidative damage caused by ROS has been frequently proposed to be associated with the pathogenesis of various diseases and health problems such as aging, arthritis, cancer, inflammation and heart diseases in human body. Consequently, antioxidants that can neutralize directly ROS attacks and terminate free radical-mediated oxidative reaction would have beneficial activities in protecting the human body from such diseases.

The development of atherosclerosis involves the infiltration of low-density lipoprotein (LDL) particles and monocytes into the vascular intima. Oxidation modified LDL, but not native LDL, has been shown like a potent chemo-attractant for circulating monocytes. ROS are also thought to mediate the injury to the myocardium brought about by ischemia and reperfusion. Various ROS scavengers have been shown to reduce this damage during myocardial ischemia/reperfusion, and some of which play clinical role in certain situations. The protective effect of certain dietary constituents is believed to be via antioxidant-mediated resistance to oxidative damage to key biomolecular, including DNA and polyunsaturated fatty acids, reducing the development of diseases, such as cancer or atherosclerosis. Panax notoginseng showed activity in decreasing total cholesterol, triglycerides and fibrinogenaemia in rats (Cicero et al., 2003).

The in vitro antioxidant activities of extract of Panax notoginseng were evaluated with different antioxidant testing systems. Panax notoginseng exhibited strong ferrous ion chelating activity and high scavenging activities against hydrogen peroxide, hydroxyl radicals, and a weak activity against superoxide anion and DPPH free radicals. These antioxidant properties of Panax notoginseng seem to be one of the reasons that they are effective in the prevention and treatment of vascular diseases (Zhao et al., 2006).

\section{Anti-inflammatory activity}

Inflammation involving the innate and adaptive immune systems is a normal response to infection. The inflammatory response is simply characterized by involving redness, swelling, heat and pain, which are classic symptoms from the three major events in inflammation. These events are vasodilatation, increasing capillary permeability and influx of phagocytes from the capillaries into the damaged tissue to attack pathogens.
Inflammation allows immune cells to quickly and efficiently travel to sites of infection, at which they can target and eradicate pathogens. However, when allowed to continue without controlled, inflammation results in autoimmune or autoinflammatory disorders, neurodegenerative disease, or cancer. Inflammation is a dynamic process with proinflammatory cytokines such as tumor necrosis factor (TNF)- $\alpha$, interleukin (IL)-1 $\beta$, and vascular endothelial growth factor (VEGF) playing central roles (Dinarello, 2010). During chronic inflammation, pro-inflammatory molecules, such as cytokines, cyclooxygenase 2 (COX-2) inducible nitric oxide synthase (iNOS), reactive oxygen species (ROS), and NF$\mathrm{kB}$ are upregulated. There are many natural and synthetic agents that are currently used in the treatment of inflammatory diseases. In spite of this, there is still a constant search for better agents to treat inflammation. There are some studies reported the antiinflammatory activity of Panax notoginseng. Panax notoginseng exerts anti-inflammatory effects related to the inhibition of neutrophil functions including degranulation, superoxide generation and leukotriene B4 production, and reduced nitric oxide NO and prostaglandin PGE2 production, which could be due to a decreased expression of iNOS and COX-2. In addition, Panax notoginseng significantly reduced mouse paw edema induced by carrageenan (Jin et al., 2007). Flower of Panax notoginseng extract significantly inhibited the productions of NO, PGE2, TNF$\alpha$ and IL-1 $\beta$ in on the LPS stimulated RAW264.7 cells. In addition, flower of Panax notoginseng extract suppressed the mRNA and protein expressions of iNOS, COX-2, TNF- $\alpha$ and IL$1 \beta$ in LPS-stimulated RAW264.7 cells. The molecular mechanism of flower of Panax notoginseng extract-mediated attenuation in RAW264.7 cells has close a relationship to suppressing the phosphorylation of MAPK molecules such as ERK1/2, JNK and p38 MAPK, and the translocation of NF- $\mathrm{kB}$ p65 subunit into nuclear (Jung et al., 2009).

Trilinolein from Panax notoginseng inhibited significantly nitric oxide (NO), tumor necrosis factor (TNF- $\alpha$ ), interleukin-1 (IL-1 $\beta$ ), and IL-6 production in a manner concentration-dependent in a model of lipopolysaccharide (LPS)stimulated mouse macrophage (RAW264.7). It also blocked the protein expression of inducible nitric oxide synthase (iNOS), cyclooxygenase-2 (COX-2), nuclear factor- $\kappa \mathrm{B}(\mathrm{NF}-\kappa \mathrm{B})$, I $\mathrm{I} \mathrm{B} \alpha$, and mitogen-activated protein kinases (MAPKs). In the antiinflammatory test, trilinolein decreased the paw edema after carrageenan administration in paw edema (Huang et al., 2014).

Notoginsenoside R1, a main bioactive component from Panax notoginseng showed capacity to alleviate the severity of dextran sulfate sodium-induced colitis in mice by decreasing the activity of myeloperoxidase, the production of cytokines, the expression of proinflammatory genes, and the phosphorylation of

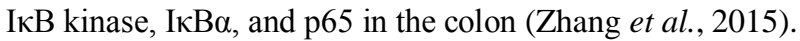

Panaxatriol saponins, the main constituents extracted from Panax notoginseng has been shown to be an effective agent in the protection against Parkinson's disease. Panaxatriol saponins provided neuroprotection against the loss of dopaminergic neurons and behavioral by including enhancing antioxidant activity, acting 
as neurotrophic factor, modulating inflammation and inhibiting mitochondria-mediated apoptosis impairment caused by 1-methyl4-phenyl-1,2,3,6-tetrahydropyridine (MPTP), a agent-induced neuronal death. Panaxatriol saponins could suppress the MPTPmediated neuronal death by increasing thioredoxin-1 expression, suppressing cyclooxygenase-2 over-expression and inhibiting mitochondria-mediated apoptosis (Luo et al., 2011).

Microglial cells activation increased neurotoxicity through the production of pro-inflammatory and cytotoxic mediators in mixed neuron-glial cultures stimulated with either toll like receptor (TLR) ligands (LPS), cytokines (TNF $\alpha$ or IFN $\gamma$ ) glutamate, Nmethyl-D-aspartate (NMDA), or $\beta$-amyloid (A $\beta$ ). Activation of microglial cells in the central nervous system may initially be intended to protect neurons; however, activation of these cells and inflammatory products derived from them has been implicated in various disease states. Effects of extract of Panax notoginseng on TLR ligand-and IFN $\gamma$-induced activation in N9 and EOC20 microglial cells lines were investigated. Panax notoginseng suppressed microglial activation by reduced expression of accessory molecules (CD40 and CD86), decreased production of inflammatory mediators (IL-6 and TNF- $\alpha$ ), and diminished release of antibacterial products (nitric oxide). Panax notoginseng and some ginsenosides such as Rb1, Rg1, or Re may be of therapeutic benefit in treating or preventing neurodegenerative diseases such as multiple sclerosis and Parkinson's disease (Beamer et al., 2012).

\section{Antithrombotic activity}

Platelets play a central role in the haemostatic process and are similarly involved in the pathological process thrombosis. They are small discoid cells derived from megakaryocytes in the bone marrow and circulate in the blood. Under normal conditions, they neither adhere to each other nor other circulating endothelial cells. When the blood vessels are damaged, subendothelial cells are exposed and platelets adhere to the disrupted surface. This adhesion is mediated by collagen and von Willebrand factor (vWF) on the subendothelial surface. Phospholipase A2 enzyme is also activated to produce a variety of prostaglandins and thromboxane.

Raw Panax notoginseng has been traditionally used as a haemostatic to arrest internal and external bleeding, reduce swelling and pain, as well as to disperse blood clots, eliminate blood stasis and promote blood circulation. Studies also showed that raw Panax notoginseng has other useful pharmacological activities on cardiovascular related diseases such as lower blood cholesterol, reduce blood pressure, treatment of coronary heart diseases, cardiac infarction and angina pectoris. Panax notoginseng (200 mg/kg i.p. x $1 \mathrm{~d}$ or $\mathrm{x} 3 \mathrm{~d}$ ) significantly inhibited abnormal increases of platelet aggregation and platelet adhesiveness in rats subjected to permanent occlusion of the middle cerebral artery. Platelet aggregation induced by ADP in vitro was also inhibited by Panax notoginseng in a concentrationrelated manner. Furthermore, Panax notoginseng could increase the fluidity of platelet membranes (Ma et al., 1998). Fibrinolysis is an integral part of the coagulation cascades. It can be regulated by the plasminogen activator (PA) and PA inhibitor (PAI-1). Impaired fibrinolysis and high plasma PAI-1 concentrations are associated with an increasing risk of cardiovascular disease. Notoginsenoside R1 has been demonstrated to upregulate tissuetype PA (tPA) without affecting urokinasetype PA (uPA) and PAI1 via increasing tPA mRNA in cultured human umbilical vein endothelial cells, while notoginsenoside R1 also could decrease PAI-1 activity via increasing complex formation between tPA and PAI-1. This saponin could also increase the production of tPA and uPA in cultured human pulmonary artery smooth muscle cells, and inhibit TNF- alpha induced PAI-1 overexpression in VSMCs by suppressing ERK and protein kinase B signaling pathways (Zhang et al., 1994, Kohler et al., 2000). Increase in plasmatic fibrinogen is regarded as a risk factor of cardiovascular disease and Panax notoginseng has been found to significantly lower the level of fibrinogen in rat models (Cicero et al., 2003).

\section{CONCLUSION}

The chemical components from Panax notoginseng have been studied and the largest class of bioactive components is saponins. Pharmacological effects of Panax notoginseng have been proven in many studies. It has shown many beneficial effects for health, including antioxidant, anti-inflammatory and antithrombotic activities. Therefore, it is important now to study how to apply Panax notoginseng to modern pharmaceutical dosage form for more convenience usage to patients.

\section{REFERENCES}

Beamer CA and Shepherd DM. Inhibition of TLR ligand- and interferon gamma-induced murine microglial activation by Panax notoginseng. J Neuroimmune Pharmacol, 2012; 7(2): 465-476.

Chan P, Thomas GN and Tomlinson B. Protective effects of trilinolein extracted from Panax notoginseng against cardiovascular disease. Acta Pharmacol Sin, 2002; 23(12): 1157-1162.

Cicero AF, Vitale G, Savino G and Arletti R. Panax notoginseng (Burk.) effects on fibrinogen and lipid plasma level in rats fed on a high-fat diet. Phytother Res, 2003; 17(2): 174-178.

Dinarello CA. Anti-inflammatory Agents: Present and Future. Cell, 2010; 140(6): 935-950.

Fan C, Song J and White CM. A Comparison of the Hemostatic Effects of Notoginseng and Yun Nan Bai Yao to Placebo Control. Journal of Herbal Pharmacotherapy, 2005; 5(2): 1-5.

Huang S-S, Deng J-S, Lin J-G, Lee C-Y and Huang G-J. AntiInflammatory Effects of Trilinolein from Panax notoginseng Through the Suppression of NF- $\mathrm{KB}$ and MAPK Expression and Proinflammatory Cytokine Expression. The American Journal of Chinese Medicine, 2014; 42(06): 1485-1506.

Jiang LA (2007). Chemical studies of Panax notoginseng and related species and evaluation of potential antiplatelet and anticoagulant effects, Doctoral dissertation

Jin UH, Park SG, Suh SJ, Kim JK, Kim DS, Moon SK, Lee YC, Park WH and Kim $\mathrm{CH}$. Inhibitory effect of Panax notoginseng on nitric oxide synthase, cyclo-oxygenase-2 and neutrophil functions. Phytother Res, 2007; 21(2): 142-148.

Jung HW, Seo UK, Kim JH, Leem KH and Park YK. Flower extract of Panax notoginseng attenuates lipopolysaccharide-induced inflammatory response via blocking of NF-kappaB signaling pathway in murine macrophages. J Ethnopharmacol, 2009; 122(2): 313-319. 
Kim D-H. Chemical Diversity of Panax ginseng, Panax quinquifolium, and Panax notoginseng. Journal of Ginseng Research, 2012; 36(1): 1-15.

Kohler HP and Grant PJ. Plasminogen-Activator Inhibitor Type 1 and Coronary Artery Disease. New England Journal of Medicine, 2000; 342(24): 1792-1801.

Lam SK and $\mathrm{Ng} \mathrm{TB}$. Isolation of a novel thermolabile heterodimeric ribonuclease with antifungal and antiproliferative activities from roots of the sanchi ginseng Panax notoginseng. Biochem Biophys Res Commun, 2001; 285(2): 419-423.

Long Y-C, Ye Y-H and Xing Q-Y. Studies on the neuroexcitotoxin $\beta$-N-oxalo-L- $\alpha, \beta$-diaminopropionic acid and its isomer $\alpha-\mathrm{N}$-oxalo-L- $\alpha$, $\beta$ diaminopropionic acid from the root of Panax species. International Journal of Peptide and Protein Research, 1996; 47(1-2): 4246.

Luo FC, Wang SD, Qi L, Song JY, Lv T and Bai J. Protective effect of panaxatriol saponins extracted from Panax notoginseng against MPTP-induced neurotoxicity in vivo. J Ethnopharmacol, 2011; 133(2): 448-453.

Ma LY and Xiao PG. Effects of Panax notoginseng saponins on platelet aggregation in rats with middle cerebral artery occlusion or in vitro and on lipid fluidity of platelet membrane. Phytotherapy Research, 1998; 12(2): 138-140.

Park W-H, Lee S-K and Kim C-H. A Korean herbal medicine, Panax notoginseng, prevents liver fibrosis and hepatic microvascular dysfunction in rats. Life Sciences, 2005; 76(15): 1675-1690.

Rhule A, Navarro S, Smith JR and Shepherd DM. Panax notoginseng attenuates LPS-induced pro-inflammatory mediators in RAW264.7 cells. J Ethnopharmacol, 2006; 106(1): 121-128.

Sun $\mathrm{H}$, Yang $\mathrm{Z}$ and Ye Y. Structure and biological activity of protopanaxatriol-type saponins from the roots of Panax notoginseng. Int Immunopharmacol, 2006; 6(1): 14-25.

Wang C-Z, McEntee E, Wicks S, Wu J-A and Yuan C-S. Phytochemical and analytical studies of Panax notoginseng (Burk.) F.H. Chen. Journal of Natural Medicines, 2006; 60(2): 97-106.

Wang D, Hong D, Koh HL, Zhang YJ, Yang CR and Hong Y. Biodiversity in cultivated Panax notoginseng populations. Acta Pharmacol Sin, 2008; 29(9): 1137-1140.

Wang J, Xu J and Zhong JB. Effect of Radix notoginseng saponins on platelet activating molecule expression and aggregation in patients with blood hyperviscosity syndrome. Zhongguo Zhong Xi Yi Jie He Za Zhi, 2004; 24(4): 312-316.
Yang X, Xiong X, Wang $\mathrm{H}$ and Wang J. Protective Effects of Panax notoginseng Saponins on Cardiovascular Diseases: A Comprehensive Overview of Experimental Studies. Evidence-Based Complementary and Alternative Medicine, 2014; 2014: 13.

Yoshikawa M, Morikawa T, Yashiro K, Murakami T and Matsuda H. Bioactive Saponins and Glycosides. XIX. Notoginseng (3): Immunological Adjuvant Activity of Notoginsenosides and Related Saponins: Structures of Notoginsenosides-L, -M, and -N from the Roots of Panax notoginseng (BURK.) F.H. Chen. Chemical and Pharmaceutical Bulletin, 2001; 49(11): 1452-1456.

Yoshikawa M, Murakami T, Ueno T, Hirokawa N, Yashiro K, Murakami N, Yamahara J, Matsuda H, Saijoh R and Tanaka O. Bioactive Saponins and Glycosides. IX. Notoginseng (2) : Structures of Five New Dammarane-Type Triterpene Oligoglycosides, Notoginsenosides-E, -G, H, -I, and -J, and a Novel Acetylenic Fatty Acid Glycoside, Notoginsenic Acid \&beta;-Sophoroside, from the Dried Root of Panax notoginseng (BURK.) F. H. Chen. Chemical and Pharmaceutical Bulletin, 1997; 45(6): 1056-1062.

Zhang HS and Wang SQ. Notoginsenoside R1 from Panax notoginseng inhibits TNF-alpha-induced PAI-1 production in human aortic smooth muscle cells. Vascul Pharmacol, 2006; 44(4): 224-230.

Zhang J, Ding L, Wang B, Ren G, Sun A, Deng C, Wei X, Mani S, Wang Z and Dou W. Notoginsenoside R1 attenuates experimental inflammatory bowel disease via pregnane $\mathrm{X}$ receptor activation. J Pharmacol Exp Ther, 2015; 352(2): 315-324.

Zhang W, Wojta J and Binder BR. Effect of notoginsenoside R1 on the synthesis of tissue-type plasminogen activator and plasminogen activator inhibitor-1 in cultured human umbilical vein endothelial cells. Arteriosclerosis, Thrombosis, and Vascular Biology, 1994; 14(7): 10401046.

Zhao G-R, Xiang Z-J, Ye T-X, Yuan Y-J and Guo Z-X. Antioxidant activities of Salvia miltiorrhiza and Panax notoginseng. Food Chemistry, 2006; 99(4): 767-774.

Zhu Y, Pettolino F, Mau SL and Bacic A. Characterization of cell wall polysaccharides from the medicinal plant Panax notoginseng. Phytochemistry, 2005; 66(9): 1067-1076.

\section{How to cite this article:}

Tung BT, Hai NT. Phytochemical and pharmacology effect of Panax notoginseng. J App Pharm Sci, 2016; 6 (08): 174-178. 